\title{
Paramètres génétiques des caractères de la portée et du poids de la mère dans le croisement de deux souches de lapin sélectionnées
}

\author{
J.M. BRUN et R. ROUVIER \\ Institut National de la Recherche Agronomique, \\ Station d'Amélioration Génétique des Animaux, B.P. 27, 31326 Castanet Tolosan Cedex, France
}

\section{Résumé}

Deux souches de lapins (INRA 1066 et 1077), originaires respectivement des races Californienne (CA) et Néo-Zélandaise Blanche (NZ) et sélectionnées sur la prolificité depuis quatre générations, sont étudiées en race pure, en croisements simples et à double étage. La taille de la portée à la naissance et au sevrage, le poids total de la portée au sevrage, le poids moyen du lapereau sevré ainsi que le poids des femelles sont analysés en termes d'effets génétiques additifs et d'hétérosis. Dans les portées de rang 1 et 2 , la souche NZ est supérieure pour les effets additifs directs sur la taille et le poids de la portée au sevrage $(+0,70$ lapereaux et $+251 \mathrm{~g}$ respectivement), ainsi que pour les effets additifs directs et maternels sur le poids de la femelle. La souche CA est supérieure pour les effets maternels sur le nombre de lapereaux nés totaux et grandsmaternels sur la viabilité des lapereaux entre la naissance et le sevrage. L'effet d'hétérosis direct sur le nombre et le poids total de lapereaux sevrés dans les portées de rang 1 et 2 est de l'ordre de $8 \%$. L'hétérosis maternel est de l'ordre de $6 \%$ sur les effectifs et poids de portée de rang 1 et 2 et de l'ordre de $3 \%$ sur l'ensemble de la carrière (7 portées). On trouve un effet d'hétérosis filial négatif sur le poids de la femelle lors de sa deuxième gestation $(-3,4 \%)$. Les résultats sont comparés avec les résultats obtenus sur les mêmes souches prises 10 ans auparavant. La différence principale concerne la mise en évidence d'un hétérosis maternel. Cela indiquerait l'intérêt d'une sélection de type récurrent et réciproque pour l'accroître. Une nouvelle expérience de croisement en cours va permettre de préciser l'évolution constatée des effets génétiques. portée.

Mots clés : lapin, effets génétiques directs, effets génétiques maternels, hétérosis, caractères de la

\section{Summary}

Genetic effects on litter traits and dam weight in crosses of two selected strains of rabbit

Two strains of rabbits (INRA 1066 and 1077), respectively originating from the breeds Californian (CA) and New-Zealand White (NZ) and selected for four generations on prolificacy have been studied in purebreeding, two and three-way crossbreeding. Litter size at birth and weaning, litter weight at weaning, average rabbit weight at weaning and doe weight have been analysed in terms of additive and non-additive genetic effects. Over the first two parities, the NZ strain has favourable direct additive effects on litter size and weight at weaning $(+0.70$ rabbits and $+251 \mathrm{~g}$ resp.), and direct and maternal additive effects on doe weight. The strain CA is the best one for maternal effects on litter size at birth (total born) and for grand-maternal effects on 
young rabbits between birth and weaning. Direct heterosis for litter size and litter weight at weaning is about $8 \%$ over parities 1 and 2 . Maternal heterosis for litter size and weight is about $6 \%$ over parities 1 and 2 and about $3 \%$ over the whole productive lifetime ( 7 parities). A negative filial heterosis effect is found on weight of does at their second gestation $(-3,4 \%)$. These results are compared with those obtained on the same strains 10 years before. The main difference relates to the appearance of maternal heterosis. This suggests the possible value of reciprocal recurrent selection for increasing it. A crossbreeding experiment in progress will allow a test of such a response.

Key words : rabbit, direct genetic effects, maternal genetic effects, heterosis, litter traits.

\section{Introduction}

Les effets génétiques qui interviennent sur les caractères de reproduction, lors du croisement entre souches de lapins, peuvent être décomposés selon le modèle de DiCKERSON (1969). En considérant ces caractères comme attributs de la portée et non de la mère (MATHERon \& MAULÉon, 1979) il s'agit de séparer les effets génétiques directs attribuables aux génotypes des lapereaux, des effets indirects, maternels et grands-maternels. On distingue également les contributions génétiques additives et non additives et, parmi celles-ci, les hétérosis directs et maternels.

Il apparaît utile de faire cette décomposition, à la fois pour une caractérisation biologique des souches, ainsi que pour l'étude des meilleures méthodes de sélection en vue du croisement. Ainsi, Brun \& Rouvier (1984), à partir d'une expérience de croisement à double étage entre les races de lapins Californienne (CA), Néo-Zélandaise Blanche (NZ) et Petite Russe (PR), réalisée de 1970 à 1972, ont estimé et discuté ces différents effets sur les caractères des portées : nombre de lapereaux dans la portée à la naissance et au sevrage, poids de la portée au sevrage. Depuis 1976, ces souches sont sélectionnées par troncature sur la valeur d'un indice du nombre de lapereaux sevrés par portée, suivant la méthode de sélection optimisée par MATHERON \& Rouvier (1977). L'utilisation des deux souches CA et NZ se fait en croisement. Il semble donc utile d'étudier l'évolution des effets génétiques précédemment mis en évidence. Pour cela, une expérience de croisement à double étage entre les deux souches $\mathrm{CA}$ et $\mathrm{NZ}$ et de croisement au stade terminal avec une troisième souche a été réalisée de 1980 à 1981. L'objet de la présente étude est d'en établir et d'en discuter les résultats.

\section{Matériel et méthode}

\section{A. Matériel biologique et protocole expérimental}

L'étude concerne les souches INRA $A 1066$ et $A 1077$, issues respectivement des races Californienne et Néo-Zélandaise Blanche. Dans le texte, elles seront désignées respectivement par les symboles CA et NZ. Les reproducteurs de l'expérience proviennent de la quatrième génération de sélection. L'expérience a eu lieu entre septembre 1979 et juillet 1981 à l'élevage expérimental INRA du Magneraud et s'est déroulée en 
deux générations successives, G1 et G2. En G1, 67 femelles de chacune des deux souches ont été accouplées selon un plan factoriel avec 6 mâles de chaque souche. En G2, 55 femelles de chacun des 4 génotypes issus de G1 ont été accouplées en croisement terminal avec des mâles de trois autres souches, de format moyen (la souche synthétique INRA 1027, sélectionnée sur la vitesse de croissance entre 28 et 77 jours, son témoin et une souche issue de la race Fauve de Bourgogne et également sélectionnée sur la vitesse de croissance). Ces femelles provenaient de la deuxième portée des femelles de $G 1$. Alors que $G 1$ a été arrêtée après la deuxième portée des femelles, $G 2$ s'est étalée sur sept parités en moyenne pour les femelles vivantes en fin d'expérience. Les conditions de conduite de l'élevage sont les suivantes : mise à la reproduction des lapines à l'âge de 120 jours; présentation au mâle 10 jours après la mise bas ; diagnostic de gestation, par palpation, 14 jours après la saillie et présentation au mâle le lendemain en cas de palpation négative ; sevrage des lapereaux à l'âge de 28 jours.

\section{B. Caractères analysés}

Sont analysés les nombres de lapereaux nés totaux, nés vivants et sevrés par portée, le poids total de la portée au sevrage et le poids moyen du lapereau sevré. Les nombres de lapereaux nés totaux et nés vivants sont analysés sur la population des portées ayant au moins un lapereau vivant à la naissance, les autres variables le sont sur la population des portées sevrées. Ces caractères sont considérés comme des attributs de la portée et non de la femelle. Nous avons également analysé le póids vif de la femelle au cours de ses portées successives, mesuré à la palpation positive.

\section{Méthode d'analyse}

La méthode utilisée pour estimer les effets génétiques sur les caractères des portées a été décrite de façon détaillée par BRUN \& Rouvier (1984) lors de l'analyse d'un plan de croisement de même structure. Les effets génétiques sont des combinaisons linéaires des valeurs génotypiques (tableau 1). Les effets génétiques additifs directs $\left(g^{l}\right)$ et l'hétérosis direct $\left(h^{I}\right)$ découlent de G1 qui permet par ailleurs d'estimer la somme $g^{M}+g^{M^{\prime}}$ des effets génétiques additifs maternels $\left(g^{M}\right)$ et grand-maternels $\left(g^{M^{\prime}}\right)$. Les effets $g^{M^{\prime}}$ et d'hétérosis maternel $h^{M}$ s'estiment à partir de G2. On obtient $g^{M}$ par différence. La première génération étant limitée aux deux premières portées de la carrière des lapines et les effets génétiques pouvant évoluer avec la parité, nous avons estimé deux séries d'effets : la première inclut l'ensemble des effets recherchés mais ne concerne que le début de carrière. Leur estimation utilise les deux premières portées de G1 et de G2. La deuxième série se limite à $g^{M^{\prime}}$ et $h^{M}$ mais concerne l'ensemble de la carrière des femelles, telle qu'elle est réalisée en $\mathrm{G} 2$.

En ce qui concerne les caractères des femelles, les effets introduits dans le modèle d'analyse génétique sont $\left(g^{F}, h^{F}, g^{I}, g^{M}\right.$ et $\left.h^{I}\right)$ où $g^{F}$ et $h^{F}$ sont des effets filiaux, respectivement additif et d'hétérosis, traduisant l'effet éventuel du génotype de la portée sur la croissance de sa mère. Ces effets sont respectivement homologues aux effets $g^{I}, h^{I}, g^{M}, g^{M^{\prime}}$ et $h^{M}$ sur les caractères des portées et leurs expressions en fonction des valeurs génotypiques figurent également au tableau 1. 


\section{Tableau 1}

Procédure d'estimation des effets génétiques sur les caractères des portées et des femelles à partir des valeurs génotypiques de $1^{r e}$ et $2^{e}$ génération

Estimation of genetic effects on litter and dam traits from genotypic values of $1^{\text {st }}$ and $2^{\text {nd }}$ generation crosses

\begin{tabular}{|c|c|c|c|c|c|c|}
\hline \multicolumn{2}{|c|}{ Caractères des } & \multicolumn{4}{|c|}{ Génotype des portées de $1^{\text {re }}$ génération } & \\
\hline portées & lapines & $\mathrm{NZ} \times \mathrm{NZ}$ & $\mathrm{CA} \times \mathrm{CA}$ & $\mathrm{NZ} \times \mathrm{CA}$ & $\mathrm{CA} \times \mathrm{NZ}$ & \\
\hline $\begin{array}{l}g_{N Z}^{l} \\
g^{M}{ }_{N Z}+g^{M}{ }_{N Z} \\
h_{N Z \times C A}^{l}\end{array}$ & $\begin{array}{l}g_{N Z}^{F} \\
g_{N Z}^{I}+g^{M}{ }_{N Z} \\
h_{N Z \times C A}^{F}\end{array}$ & $\begin{array}{r}1 / 2 \\
0 \\
-1 / 2 \\
\end{array}$ & $\begin{array}{c}-1 / 2 \\
0 \\
-1 / 2 \\
\end{array}$ & $\begin{array}{r}1 / 2 \\
-1 / 2 \\
1 / 2 \\
\end{array}$ & $\begin{array}{r}-1 / 2 \\
1 / 2 \\
1 / 2 \\
\end{array}$ & \\
\hline \multicolumn{2}{|c|}{ Caractères des } & \multicolumn{4}{|c|}{ Génotype des portées de $2^{e}$ génération } & \multirow{2}{*}{$\begin{array}{l}\text { Estimée }{ }^{+} \text {de } \\
g^{M}{ }_{N Z}+g^{M^{\prime}}{ }_{N Z}\end{array}$} \\
\hline portées & lapines & $\mathrm{T} \times(\mathrm{NZ} \times \mathrm{NZ})$ & $\mathrm{T} \times(\mathrm{CA} \times \mathrm{CA})$ & $\mathrm{T} \times(\mathrm{NZ} \times \mathrm{CA})$ & $\mathrm{T} \times(\mathrm{CA} \times \mathrm{NZ})$ & \\
\hline $\begin{array}{l}g^{M}{ }_{N Z} \\
g^{M^{\prime}}{ }_{N Z} \\
h^{M}{ }_{N Z \times C A}\end{array}$ & $\begin{array}{l}g_{N Z}^{\prime} \\
g_{N Z}^{M}{ }_{N Z} \\
h_{N Z \times C A}^{l}\end{array}$ & $\begin{array}{c}0 \\
0 \\
-1 / 2\end{array}$ & $\begin{array}{c}0 \\
0 \\
-1 / 2\end{array}$ & $\begin{array}{r}1 / 2 \\
-1 / 2 \\
1 / 2\end{array}$ & $\begin{array}{r}-1 / 2 \\
1 / 2 \\
1 / 2\end{array}$ & $\begin{array}{l}1 \\
0 \\
0\end{array}$ \\
\hline
\end{tabular}

Quel que soit l'effet additif, $g_{C A}=-g_{N Z}$. (For any additive effect, $g_{C A}=-g_{N Z}$.)

+ Estimée résultant de la $1^{\text {rc }}$ génération et valant pour les caractères des portées. Pour les caractères des femelles, il faut lire $g^{J}+g^{M}$. (Estimate from the $1^{s t}$ generation, valid for litter traits. For dam traits, substitute $g^{\prime}+g^{M}$.)

En G2, une analyse de variance incluant l'effet du génotype du père de la portée indique que cet effet n'est pas significatif et ne présente pas d'interaction avec l'effet du génotype de la mère. Donc les valeurs génotypiques ont été estimées par analyse de variance avec les effets fixés "génotype de la portée " (en G1) ou "génotype de la mère » (en G2), parité et leur interaction. Les calculs ont été réalisés à l'aide de logiciels de la programmathèque AMANCE sur le MINI 6 du centre de calcul de l'INRA de Toulouse.

\section{Résultats}

A. Le début de la carrière: parités 1 et 2

\section{Résultats globaux}

Le tableau 2 donne les estimées des valeurs des génotypes de G1 et de G2. En G1, l'effet du génotype de la portée n'est pas significatif sur les tailles de portées à la naissance mais le devient sur les tailles et poids de portée au sevrage. Cela est lié à la forte mortalité des lapereaux de génotype $\mathrm{CA} \times \mathrm{CA}$ entre la naissance et le sevrage. L'interaction entre les effets génotype de la portée et parité n'est significative sur aucun 


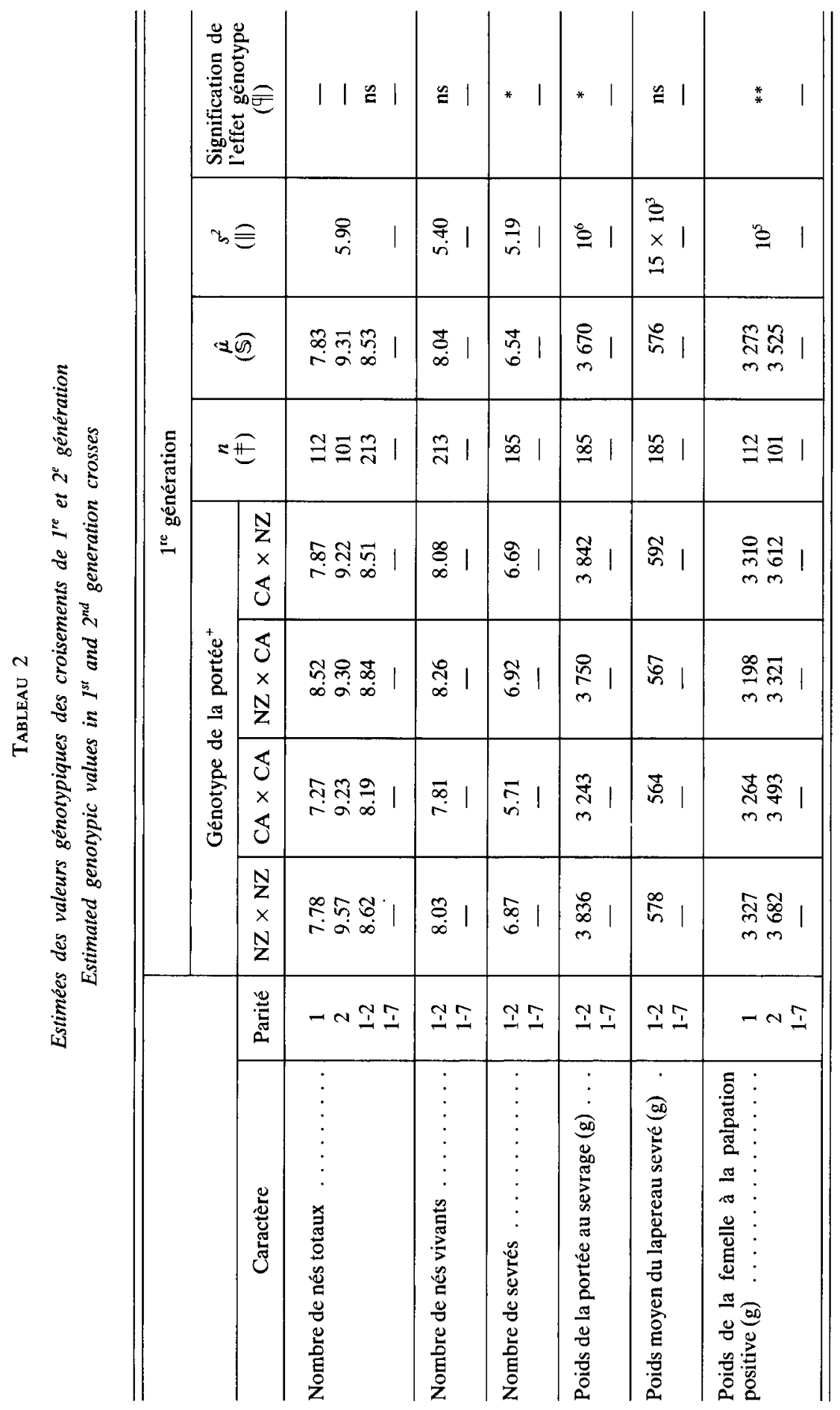




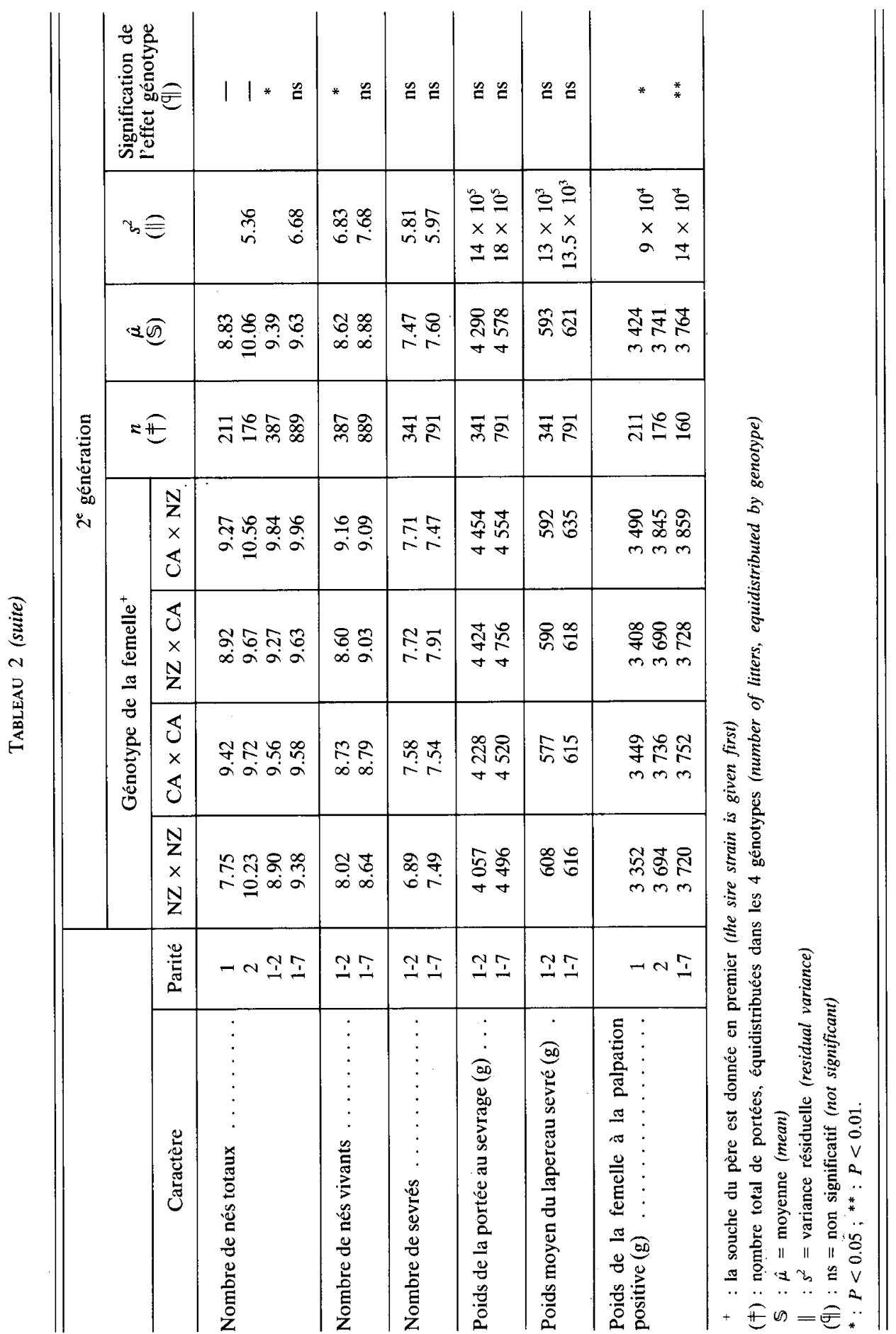


des caractères analysés. En G2, il existe des différences significatives entre génotypes maternels pour les nombres de nés totaux et nés vivants par portée. Ces différences n'existent plus au sevrage. On observe une interaction significative entre le génotype de la femelle et la parité sur le nombre de nés totaux par portée, ce qui amène à analyser ce caractère dans les portées de rang 1 et 2 comme deux caractères différents.

\section{Effets génétiques}

a) Caractères des portées

On remarque (tableau 3) la supériorité de la souche NZ pour les effets additifs directs sur les tailles et poids de portées au sevrage, quelle que soit la parité. En portée 1 , ces effets se manifestent dès la naissance, sur le nombre de nés totaux. En ce qui concerne les effets maternels, la souche CA montre une supériorité significative au seuil de $5 \%$ sur le nombre de nés totaux $\left(g^{M}{ }_{N Z}=-0,46\right)$. Le fait que cette supériorité disparaisse au sevrage $\left(g^{M}{ }_{N Z}=-0,12\right)$ indique l'existence d'effets maternels en sens opposé sur la viabilité des lapereaux entre la naissance et le sevrage. Les effets grandmaternels apparaissent en opposition aux effets maternels sur le nombre de nés totaux en portée 2. Comme les effets additifs directs, les hétérosis sur les effets génétiques directs sont significatifs surtout au sevrage sur la taille et le poids de portée $\left(h^{I}=0,52\right.$ et $256 \mathrm{~g}$ respectivement), mais également en portée 1 sur le nombre de nés totaux. On note de l'hétérosis maternel sur toutes les variables étudiées, sauf le nombre de nés totaux en portée 2 et le poids moyen du lapereau sevré. Une analyse par parité montre qu'il n'y a pas d'hétérosis maternel sur la taille et le poids de portée au sevrage en portée 2 .

\section{b) Poids des femelles à la palpation}

L'effet génétique additif filial $g^{F}$ n'est pas significatif dans les cas où il a pu être calculé. On note globalement des effets additifs directs et maternels positifs de la souche NZ (non significatif uniquement pour $g^{I}{ }_{N Z}$ en première palpation). L'hétérosis filial n'est pas significatif en première palpation, il est significativement négatif en deuxième palpation. L'hétérosis direct est également significatif en deuxième palpation, non significatif en première palpation.

\section{B. L'ensemble de la carrière : parités 1 à 7 (tableau 3)}

\section{1) Résultats globaux}

Les différents génotypes de femelles ne présentent plus, sur l'ensemble de la carrière, de différences significatives sur les composantes de la productivité numérique et pondérale à la naissance et au sevrage. Seules sont significatives les différences de poids des femelles à la palpation positive. Il n'y a pas d'interaction entre le génotype de la mère et la parité. 


\section{TABLEAU 3}

Estimées des effets génétiques sur les caractères des portées et des femelles Estimated genetic effects for the litter and the dam traits

\begin{tabular}{|c|c|c|c|c|c|c|c|c|}
\hline \multicolumn{9}{|c|}{ Caractères des portées } \\
\hline \multirow{2}{*}{ Caractère } & \multirow{2}{*}{ Parité } & \multirow{2}{*}{$g_{N Z}^{g}$} & \multirow{2}{*}{$g^{M}{ }_{N Z}$} & \multirow{2}{*}{$g^{M^{\prime}}{ }_{N Z}$} & \multicolumn{2}{|c|}{$h_{C A \times N Z}^{\prime}$} & \multicolumn{2}{|c|}{$h^{M}{ }_{C A \times N Z}$} \\
\hline & & & & & abs. & $\%$ & abs. & $\%$ \\
\hline Nés totaux & $\begin{array}{c}1 \\
2 \\
1 \text { à } 2 \\
1 \text { à } 7\end{array}$ & $\begin{array}{l}0.58^{*} \\
0.21 \\
0.38 \\
-\end{array}$ & $\begin{array}{c}-0.48^{*} \\
-0.49^{*} \\
-0.46^{*} \\
-\end{array}$ & $\begin{array}{l}0.16 \\
0.45^{* *} \\
0.29^{*} \\
0.16\end{array}$ & $\begin{array}{c}0.70^{*} \\
-0.14 \\
0.27 \\
-\end{array}$ & 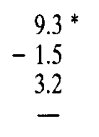 & $\begin{array}{l}0.50^{*} \\
0.14 \\
0.32 \\
0.31\end{array}$ & $\begin{array}{l}5,8^{*} \\
1.4 \\
3.5 \\
3.2^{*}\end{array}$ \\
\hline Nés vivants & $\begin{array}{l}1 \text { à } 2 \\
1 \text { à } 7\end{array}$ & 0.20 & $\begin{array}{c}-0.37 \\
-\end{array}$ & $\begin{array}{l}0.28 \\
0.03\end{array}$ & $\begin{array}{l}0.25 \\
-\end{array}$ & $\begin{array}{l}3.2 \\
-\end{array}$ & $\begin{array}{l}0.50^{*} \\
0.34^{*}\end{array}$ & $\begin{array}{l}6.0^{*} \\
4.0^{*}\end{array}$ \\
\hline Sevrés .............. & $\begin{array}{l}1 \text { à } 2 \\
1 \text { à } 7\end{array}$ & $0.70^{*}$ & -0.12 & $\begin{aligned} & 0.00 \\
- & 0.22 *\end{aligned}$ & $\begin{array}{l}0.52^{*} \\
-\end{array}$ & $\frac{8.3^{*}}{-}$ & $\begin{array}{l}0.49^{*} \\
0.18\end{array}$ & $\begin{array}{l}6.8^{*} \\
2.4 \\
\end{array}$ \\
\hline Poids de la portée sevrée (g) & $\begin{array}{l}1 \text { à } 2 \\
1 \text { à } 7\end{array}$ & $\begin{array}{l}251^{*} \\
-\end{array}$ & $\stackrel{30}{-}$ & $\begin{array}{r}15 \\
-101\end{array}$ & $\begin{array}{l}256^{*} \\
-\end{array}$ & $\begin{array}{l}7.2^{*} \\
-\end{array}$ & $\begin{array}{l}296^{*} \\
147^{*}\end{array}$ & $\begin{array}{l}6.9^{*} \\
3,2 *\end{array}$ \\
\hline $\begin{array}{l}\text { Poids moyen du lapereau } \\
\text { sevré }(\mathrm{g}) \ldots \ldots \ldots \ldots \ldots\end{array}$ & $\begin{array}{l}1 \text { à } 2 \\
1 \text { à } 7\end{array}$ & $\begin{array}{l}-5 \\
-\end{array}$ & $\frac{13}{-}$ & $\begin{array}{l}1 \\
8.5\end{array}$ & $\begin{array}{r}9 \\
-\end{array}$ & 1.5 & $\begin{array}{r}-1 \\
11\end{array}$ & $\begin{array}{l}0.0 \\
1.8\end{array}$ \\
\hline \multicolumn{9}{|c|}{ Caractères des femelles } \\
\hline \multirow{2}{*}{ Caractère } & \multirow{2}{*}{ Parité } & \multirow{2}{*}{$g_{N Z}^{F}$} & \multirow{2}{*}{$g_{N Z}^{I}$} & \multirow{2}{*}{$g^{M}{ }_{N Z}$} & \multicolumn{2}{|c|}{$h_{C A \times N Z}^{F}$} & \multicolumn{2}{|c|}{$h_{C A \times N Z}^{l}$} \\
\hline & & & & & abs. & $\%$ & abs. & $\%$ \\
\hline Poids à la palpation positive & $\begin{array}{c}1 \\
2 \\
1 \text { à } 7\end{array}$ & $\begin{array}{r}-24 \\
-50 \\
-\end{array}$ & $\begin{array}{l}15 \\
68 * \\
-\end{array}$ & $\begin{array}{l}41^{*} \\
77^{* *} \\
65^{* *}\end{array}$ & $\begin{array}{c}-42 \\
-121^{*} \\
-\end{array}$ & $\begin{array}{c}-1.3 \\
-3.4^{*} \\
-\end{array}$ & $\begin{array}{l}48 \\
52 * \\
57\end{array}$ & $\begin{array}{l}1.5 \\
1.5^{*} \\
1.5\end{array}$ \\
\hline \multicolumn{9}{|c|}{$\begin{array}{l}g^{F}, g^{I}, g^{M}, g^{M}=\text { effet génétique additif respectivement filial, direct, maternel et grand-maternel (additive geneti } \\
\text { effect resp. filial, direct, maternal and grand-maternal). }\end{array}$} \\
\hline \multicolumn{9}{|c|}{$\begin{array}{l}\text { Pour tout effet additif, } g_{C A}=-g_{N Z} \text { (for any additive effect, } \mathrm{g}_{\mathrm{CA}}=-\mathrm{g}_{\mathrm{NZ}} \text { ). } \\
h^{F}, h^{h}, h^{M}=\text { hétérosis respectivement filial, direct et maternel (filial, direct and maternal heterosis). } \\
{ }^{*}: P<0.05 ;{ }^{* *}: P<0.01 \\
\text { abs. = valeur absolue (absolute value). }\end{array}$} \\
\hline
\end{tabular}

\section{2) Effets génétiques}

Les effets grand-maternels ne sont pas significatifs pour les nombres de nés totaux et nés vivants par portée. Pour la taille de portée sevrée, la souche NZ a un effet grand-maternel significativement négatif $\left(g^{M^{\prime}}{ }_{N Z}=-0,22\right)$, ce qui traduit un effet grandmaternel défavorable sur la viabilité des lapereaux. Cet effet se manifestait déjà en début de carrière. L'hétérosis sur les effets maternels est significatif sur le nombre de lapereaux nés totaux, nés vivants, à la limite de la signification sur le nombre de lapereaux sevrés mais significatifs sur le poids des lapereaux sevrés. Sur le poids des femelles à la palpation positive, on enregistre, comme en première et deuxième 
palpation positive, un effet maternel positif de la souche NZ. L'hétérosis direct sur ce caractère conserve la valeur de $1,5 \%$ (non significative) observée à la mise en reproduction.

\section{Discussion}

Ces deux mêmes souches ont déjà fait l'objet d'une analyse génétique semblable à l'occasion d'une expérimentation réalisée 10 ans auparavant (1970-1972), qui incluait de plus la souche A1088 d'origine Petite Russe. Le caractère nouveau considéré est ici le poids à la palpation positive de la lapine. La discussion va d'abord porter sur celui-ci. La souche NZ se caractérise par un effet génétique additif direct sur le poids à la palpation positive pour la deuxième gestation plus élevé que celui de la souche CA. Cela traduit un potentiel génétique de croissance plus élevé entre la première et la deuxième gestation. L'effet génétique additif filial sur le poids à la palpation positive n'est pas significatif, ce qui est logique pour la première palpation et également pour la deuxième. En effet, il n'y a pas d'effet génétique additif direct de la souche NZ $\left(g_{N Z}^{I}\right)$ significatif sur le poids de la portée sevrée de rang 1. Par contre l'hétérosis filial significativement négatif à la deuxième palpation sur le poids de la femelle peut être mis en relation avec un effet d'hétérosis direct sur le poids au sevrage de la première portée. Cela indique chez les lapines qui élèvent des lapereaux croisés une compétition entre la poursuite de la croissance corporelle d'une part, la reproduction et l'allaitement d'autre part au profit de ceux-ci.

En ce qui concerne les caractères des portées, le tableau 4 permet de comparer les résultats de 1970 et de 1980 pour le nombre de lapereaux nés vivants par portée et de lapereaux sevrés par portée. Pour certains effets $\left(g^{I}, h^{I}\right.$ et $\left.g^{M}\right)$, les deux estimations ne sont pas strictement comparables puisque les effets estimés en 1980 ne le sont que sur les deux premières portées de la carrière : l'influence de la portée 1 où l'expression des caractères de productivité numérique est particulière (Hulot \& MATHERON $_{n}$ 1981) n'autorise pas une comparaison rigoureuse. On note cependant une constance de l'effet maternel sur le nombre de lapereaux nés par portée bien qu'ici, significatif pour le nombre de nés totaux, il ne le soit pas pour le nombre de nés vivants. L'effet maternel positif de la souche CA sur cette variable avait été interprété comme une supériorité de cette souche pour le taux d'ovulation. Cela fut confirmé par la mesure directe de ce caractère dans les deux souches, révélant une différence de deux ovules pondus en faveur de la souche CA (Hulot \& MATHERon, 1981), quelles que soient la parité (1 à 3) et la saison. L'hétérosis direct a la même signification entre les deux expériences : non significatif à la naissance, significatif au sevrage $(8,3 \%)$. Cela confirme l'existence d'un hétérosis direct sur la viabilité naissance-sevrage des lapereaux. Il apparaît en 1980 une différence importante d'effet direct sur la viabilité naissance-sevrage en faveur de la souche $\mathrm{NZ}$; cette différence découle algébriquement de la très forte mortalité des lapereaux Californiens purs (37\% des lapereaux nés vivants, soit plus du double de la mortalité en souche NZ). Il reste à étudier dans les générations ultérieures si cette plus faible viabilité des lapereaux Californiens purs se confirme ou non et, si oui, à rechercher des explications dans l'évolution de la consanguinité des souches.

Pour les effets $g^{M^{\prime}}$ et $h^{M}$ estimés sur l'ensemble de la carrière, la comparaison entre années est permise. On observe deux changements : le premier est que l'effet génétique additif grand-maternel de la souche $\mathrm{NZ}$ sur le nombre de lapereaux sevrés par portée, 
qui était significativement positif en 1970 est significativement négatif en 1980. Cet effet grand-maternel n'étant pas significatif sur le nombre de nés vivants par portée, il représente donc un effet sur la viabilité naissance-sevrage des lapereaux. La différence de tailles de portée d'origine pour les deux types génétiques $\mathrm{CA} \times \mathrm{NZ}$ et $\mathrm{NZ} \times \mathrm{CA}$, qui est dans le même sens en 1970 et en 1980, ne permet pas d'expliquer ce changement. Par contre les souches CA et NZ sélectionnées depuis 1974 ont évolué sous l'effet de cette sélection (Matheron, 1982 ; Matheron \& Poujardieu, 1984). Ce changement pourrait donc être lié à une modification de la structure génétique des deux souches.

\section{TABLEAU 4}

Comparaison des effets génétiques estimés sur les mêmes souches à 10 ans d'intervalle Comparison of the genetic effects between 2 experiments performed at an interval of 10 years

\begin{tabular}{l|c|c|c|c|c|c|c|c|c|c}
\hline & \multicolumn{2}{|c|}{$g^{I}{ }_{N Z}$} & \multicolumn{2}{|c|}{$g^{M}{ }_{N Z}$} & \multicolumn{2}{c|}{$g^{M^{\prime}}$ NZ } & \multicolumn{2}{c|}{$h^{I}(\%)$} & \multicolumn{2}{c}{$h^{M}(\%)$} \\
\cline { 2 - 12 } & 1970 & 1980 & 1970 & 1980 & 1970 & 1980 & 1970 & 1980 & 1970 & 1980 \\
\hline $\begin{array}{l}\text { Nombre de lapereaux } \\
\text { nés vivants par portée }\end{array}$ & 0.08 & 0.20 & -0.36 & -0.37 & 0.20 & 0.03 & 5.3 & 3.2 & 0.0 & $4.0^{*}$ \\
\hline $\begin{array}{l}\text { Nombre de lapereaux } \\
\text { sevrés par portée ... }\end{array}$ & 0.12 & $0.70^{*}$ & $-0.98^{*}$ & -0.12 & $0.66^{*}$ & $-0.22^{*}$ & $10.7^{*}$ & $8.3^{*}$ & -4.2 & 2.4 \\
\hline
\end{tabular}

Les résultats de l'expérience de 1970 diffèrent de ceux qui ont été publiés (BRUN \& ROUVIER, 1984) car ils ont été recalculés dans le cadre d'un croisement à 2 souches au lieu de 3 .

The results of the 1970 experiment differ from those published (BRUN \& ROUVIER, 1984) because they have been recalculated with a 2 strains model instead of 3 .

Le deuxième changement est l'apparition d'un effet d'hétérosis maternel sur la taille de portée à la naissance, au sevrage (en début de carrière mais pas sur l'ensemble de la carrière), et sur le poids de portéè sevrée. Ce résultat ne concorde pas avec les résultats généraux de la littérature, portant sur d'autres espèces, concernant l'évolution de l'hétérosis entre deux populations soumises à une sélection intra population. Dans toutes ces expériences, le profil de la réponse mesurée en croisement est parallèle à celui des populations parentales (BRUN, 1985). C'est dire que l'hétérosis y reste stable en valeur absolue par sélection intra population. Cependant, Hulot \& MATHERoN (1979) ont mis en évidence un hétérosis maternel de $28 \%$ sur le nombre d'embryons à 16 jours de gestation en parité 2, lors d'une expérience de croisement réalisée en 1974 entre ces deux souches. L'hétérosis maternel mis en évidence dans l'expérience actuelle est relativement faible (3 à $4 \%$ ). Il peut s'expliquer par une modification de la structure génétique des souches et sera intéressant à exploiter s'il se confirme.

\section{Conclusion}

Cette expérience a permis d'estimer à nouveau les effets génétiques sur les caractères des portées et sur le poids de la lapine dans le croisement des deux souches $\mathrm{CA}$ et $\mathrm{NZ}$ après 10 années de sélection intra population. L'on trouve une supériorité 
de l'effet maternel de la souche CA sur la taille de portée à la naissance qui confirme sa supériorité pour le taux d'ovulation. L'on retrouve l'existence d'un hétérosis direct sur la viabilité naissance-sevrage des lapereaux. Par contre l'effet grand-maternel sur la taille de portée sevrée précédemment trouvé favorable chez la NZ n'est pas confirmé puisqu'il est trouvé favorable chez la CA. De plus cette expérience met en évidence un effet d'hétérosis maternel sur les tailles de portée. L'hétérosis filial négatif sur le poids de la lapine à la deuxième palpation positive, c'est-à-dire pendant l'allaitement de la première portée et le début de la deuxième gestation, traduit une orientation du métabolisme préférentiellement pour satisfaire les besoins de reproduction, dans cette phase de croissance corporelle de la mère.

Une nouvelle expérience de croisement en cours va permettre de mieux connaître l'évolution de ces effets génétiques. Cette nouvelle expérience utilise les souches CA et $\mathrm{NZ}$ prises à la $14^{\mathrm{e}}$ génération de sélection. La présence d'une troisième souche, de même origine raciale que la souche $\mathrm{NZ}$ mais maintenue sans sélection, devrait permettre d'analyser l'effet de la sélection sur les paramètres du croisement. Si l'hétérosis maternel se confirme, il indiquera d'une part l'intérêt de l'utilisation de la lapine croisée, d'autre part celui d'une sélection de type récurrent et réciproque pour l'accroître.

Reçu le 10 août 1987.

Accepté le 16 novembre 1987.

\section{Références bibliographiques}

BRUN J.M., 1985. Interactions géniteur $\times$ population des partenaires. 3. Synthèse bibliographique. Génét. Sél. Evol., 17, 561-578.

BRUN J.M., Rouvier R., 1984. Effets génétiques sur les caractères des portées issues de trois souches de lapins utilisées en croisement. Génét. Sél. Evol., 16, 367-384.

DicKerson G.E., 1969. Experimental approaches in utilising breed resources. Anim. Breed. Abstr., 37, 191-202.

Hulot F., Matheron G., 1979. Analyse des variations génétiques entre trois races de lapins de la taille de portée et de ses composantes biologiques en saillie post-partum. Ann. Génét. Sél. Anim., 11, 53-77.

Hulot F., Matheron G., 1981. Effet du génotype, de l'âge et de la saison sur les composantes de la reproduction chez la lapine. Ann. Génét. Sél. Anim., 13, 131-150.

Matheron G., 1982. Genetic and selection of litter size in rabbit. In : $2^{\text {nd }}$ world congress of genetics applied to livestock production, Madrid, October 4-8, 1982, vol. 6, 481-493, Editorial Garsi, Madrid.

Matheron G., Mauléon P., 1979. Mise en évidence de l'action conjointe des effets directs, maternels et grand-maternels sur la taille de portée. Bull. Tech. Dép. Génét. Anim., 29-30, 232-263, CNRZ, Jouy-en-Josas.

Matheron G., Poujardieu B., 1984. Expérience de sélection de la taille de portée chez le lapin. In: Proceedings of the 3rd World Rabbit Congress, Rome, April 4-8, 1984, vol. 1, 66-78, World Rabbit Science Association, Rome.

Matheron G., Rouvier R., 1977. Optimisation du progrès génétique sur la prolificité chez le lapin. Ann. Génét. Sél. Anim., 9, 393-405. 